\title{
CRYSTALLIZATION, STRUCTURE AND DYNAMICS OF THE PROTON- TRANSLOCATING P-TYPE ATPase
}

\author{
GENE A. SCARBOROUGH* \\ Department of Pharmacology, University of North Carolina, School of Medicine, Chapel Hill, NC 27599, USA \\ *e-mail: gas@med.unc.edu
}

Accepted 19 October; published on WWW 13 December 1999

\begin{abstract}
Summary
Large single three-dimensional crystals of the dodecylmaltoside complex of the Neurospora crassa plasma membrane $\mathrm{H}^{+}$-ATPase $\left(\mathrm{H}^{+}\right.$P-ATPase) can be grown in polyethylene-glycol-containing solutions optimized for moderate supersaturation of both the protein surfaces and detergent micellar region. Large two-dimensional $\mathbf{H}^{+} \mathbf{P}$ ATPase crystals also grow on the surface of such mixtures and on carbon films located at such surfaces. Electron crystallographic analysis of the two-dimensional crystals grown on carbon films has recently elucidated the structure of the $\mathrm{H}^{+} \mathrm{P}$-ATPase at a resolution of $0.8 \mathrm{~nm}$ in the membrane plane. The two-dimensional crystals comprise two offset layers of ring-shaped ATPase hexamers with their exocytoplasmic surfaces face to face. Side-to-side interactions between the cytoplasmic regions of the hexamers in each layer can be seen, and an interaction between identical exocytoplasmic loops in opposing hexamer layers holds the two layers together. Detergent rings around the membrane-embedded region of the hexamers are clearly visible, and detergent-detergent interactions between the rings are also apparent. The crystal packing forces thus comprise both protein-protein and detergent-detergent interactions, supporting the validity of the original crystallization strategy. Ten transmembrane helices in each ATPase monomer are welldefined in the structure map. They are all relatively

straight, closely packed, moderately tilted at various angles with respect to a plane normal to the membrane surface and average approximately $3.5 \mathrm{~nm}$ in length. The transmembrane helix region is connected in at least three places to the larger cytoplasmic region, which comprises several discrete domains separated by relatively wide, deep clefts. Previous work has shown that the $\mathrm{H}^{+} \mathrm{P}$-ATPase undergoes substantial conformational changes during its catalytic cycle that are not changes in secondary structure. Importantly, the results of hydrogen/deuterium exchange experiments indicate that these conformational changes are probably rigid-body interdomain movements that lead to cleft closure. When interpreted within the framework of established principles of enzyme catalysis, this information on the structure and dynamics of the $\mathrm{H}^{+}$P-ATPase molecule provides the basis of a rational model for the sequence of events that occurs as the ATPase proceeds through its transport cycle. The forces that drive the sequence can also be clearly stipulated. However, an understanding of the molecular mechanism of ion transport catalyzed by the $\mathrm{H}^{+} \mathrm{P}$-ATPase awaits an atomic resolution structure.

Key words: membrane, transport, protein, crystallization, P-type ATPase, proton pump, structure, conformational change, molecular dynamics.
\end{abstract}

\section{Introduction}

Ion-translocating ATPases are essential cellular energy converters that transduce the chemical energy of ATP hydrolysis into transmembrane ionic electrochemical potential differences. These ion gradients in turn power diverse cellular functions including absorption, secretion, transmembrane signalling, nerve impulse transmission, excitation/contraction coupling, growth and differentiation. A major class of iontranslocating ATPase is the P-type ATPase family, with members throughout the phylogenetic tree (Stangeland et al., 1997). Members of this family share numerous regions of amino acid sequence homology and a molecular mechanism that involves the transient formation of a high-energy aspartyl- phosphoryl-enzyme intermediate, which is the basis for the term P-type ATPase (Pedersen and Carafoli, 1987). The phosphoryl-enzyme intermediate is undoubtedly intimately involved in the molecular mechanism of ion transport catalyzed by these enzymes. A primary goal remaining in the field of membrane transport is an elucidation of the molecular mechanism by which the P-type ATPases drive ions across membranes at the expense of ATP hydrolysis. An enormous amount of work has been expended to this end (Lutsenko and Kaplan, 1995; Moller et al., 1996), but progress has been slow because of the lack of information about the structure of these enzymes. The P-type ATPase of primary interest in my 
laboratory is the proton-translocating ATPase from the plasma membrane of Neurospora crassa, and in the past few years we have made significant progress towards elucidating the structure of this enzyme. In this article, this progress is briefly described. It is then shown how this new structural information, when taken together with the biochemical information that we have accumulated for the $\mathrm{H}^{+} \mathrm{P}$-ATPase, can provide a reasonable description of the molecular dynamics of this transporter during its ion-pumping cycle.

\section{Crystallization of the $\mathrm{H}^{+}$P-ATPase}

The fundamental advance that made possible much of our recent progress with the $\mathrm{H}^{+} \mathrm{P}$-ATPase was the development of a new approach to the crystallization of the detergent complexes of integral membrane proteins, utilizing the ATPase as our first experimental object (Scarborough, 1994). The standard approach to crystallizing soluble proteins involves an empirical search for an appropriate precipitant that will render the protein solution mildly supersaturated, from which crystals will form as protein-protein interactions replace solvent-protein interactions. However, when this approach is tried with detergent complexes of membrane proteins, crystals are rarely produced. It seemed possible that the chimeric nature of membrane protein-detergent complexes is a fundamental problem that often precludes their crystallization using the standard approach. That is, detergent complexes of integral membrane proteins are chimeras, with conventional protein surfaces made up of the standard polypeptide functional groups and with a detergent micellar collar surrounding the part of the protein normally embedded in the lipid bilayer. And whereas it should be relatively straightforward to find conditions empirically that render the protein part of a membrane protein-detergent complex mildly supersaturated, it is unlikely that those conditions would also render the detergent micellar collar suitably insoluble. It was thus reasoned that a key to crystallizing membrane-protein detergent complexes may be the attainment of conditions in which the protein surfaces of the chimera are moderately supersaturated, encouraging protein-protein interactions, and in which the the detergent micellar collar is also at or near its solubility limit so that detergent-detergent interactions are promoted as well. These considerations suggested that the conventional random search for suitable precipitants was not likely to be a very successful approach for crystallizing the ATPase, which is purified as its dodecylmaltoside (DDM) complex, and numerous unsuccessful attempts to crystallize it in this way lent support to this conclusion.

It was therefore decided to approach the problem more systematically in an attempt to find conditions in which the extents of supersaturation of the protein surfaces of the ATPase and the detergent micellar collar were appropriately matched. The first step was to screen a variety of the common protein precipitants for those that were able to induce the aggregation of pure DDM micelles under conditions where the ATPase is most stable. For those that did, such as polyethylene glycols
$400,600,1000,2000$ and 4000, their concentrationdependence was determined. It was hoped that the concentration at which each precipitant induced insolubility of the pure DDM micelles might be close to the concentration at which it would induce insolubility of the detergent micellar collar of the DDM-ATPase complex. Crystallization trials of the ATPase-DDM complex were then set up near these critical precipitant concentrations, and ATPase crystals were obtained for four different precipitants. Subsequent optimization of the initial crystallization conditions has yielded large, single crystals of the ATPase that grow to approximately $400 \mu \mathrm{m} \times$ $400 \mu \mathrm{m} \times 150 \mu \mathrm{m}$ in size and occasionally diffract X-rays to better than $0.4 \mathrm{~nm}$ resolution using synchrotron radiation. An $\mathrm{X}$-ray diffraction analysis of the $\mathrm{H}^{+} \mathrm{P}$-ATPase crystals is in progress, and although these crystals have presented us with numerous technical problems, presumably because of their detergent content, we are hopeful that they may eventually yield a structure at atomic resolution.

Electron microscopy of three-dimensional crystals has often been used to obtain intermediate-level resolution images of protein molecules, and this information can sometimes aid in the interpretation of higher-resolution X-ray diffraction data (McPherson, 1982). For this reason, we decided to initiate an exploration of the $\mathrm{H}^{+}$P-ATPase crystals using electron microscopic techniques. To facilitate these studies, the ATPase crystallization procedure was adapted for the production of thin microcrystals of the enzyme (G. A. Scarborough, unpublished experiments). In addition to providing microcrystals of the ATPase, these early studies clearly showed the predilection of the ATPase for crystallizing on surfaces, as crystals were found in large numbers growing on glass coverslips during light microscopy. In a subsequent electron microscopic investigation of the three-dimensional microcrystals, it was then discovered that large, two-dimensional crystals also grow in the microcrystallization drops at the air-water interface (Cyrklaff et al., 1995). Image processing of selected images of the surface crystals yielded a projection map of the ATPase at $1.03 \mathrm{~nm}(10.3 \AA$ ) resolution (Cyrklaff et al., 1995), and subsequent electron crystallographic analysis of twodimensional crystals grown directly on carbon-coated electron microscope grids (Auer et al., 1999) yielded a threedimensional map of the ATPase structure at a resolution of $0.8 \mathrm{~nm}$ in the membrane plane (Auer et al., 1998).

\section{Structure of the $\mathrm{H}^{+} \mathbf{P}$-ATPase}

Fig. 1A shows a cross section of the $\mathrm{H}^{+} \mathrm{P}$-ATPase structure map cut perpendicular to the two-dimensional crystal and membrane plane. The crystals consist of two layers of ATPase hexamers, which are the fundamental crystallizing unit (Cyrklaff et al., 1995; Chadwick et al., 1987). The hexamer layers are separated by a narrow region of low density and are related to one another by twofold symmetry. Within each layer, regions of tightly packed rod-shaped densities, characteristic of transmembrane $\alpha$-helices at this level of resolution and perpendicular to the membrane plane, define the membrane- 

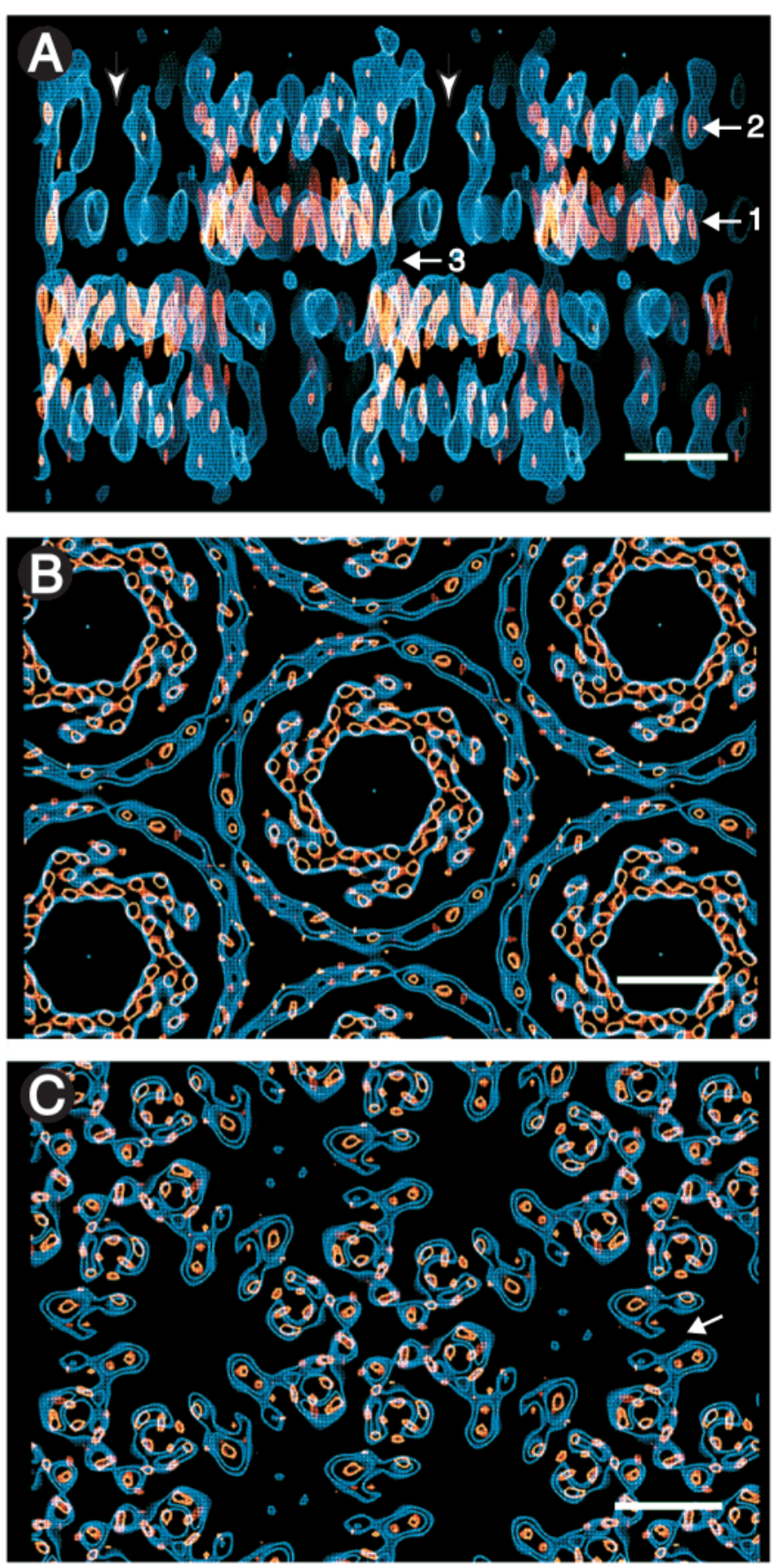

spanning domain of the ATPase (arrow 1). The large cytoplasmic regions of the $\mathrm{H}^{+} \mathrm{P}$-ATPase (arrow 2) extend from the membrane domains towards each of the two-dimensional crystal surfaces. This view also shows the main interconnection between the layers (arrow 3), which occurs via a loop of the polypeptide chain that extends from two transmembrane helices approximately $0.6 \mathrm{~nm}$ beyond the exocytoplasmic surface and makes contact with an identical loop from a corresponding monomer in the other layer. There are three of these contacts for each ATPase hexamer.

Fig. 1B shows a section cut parallel to the crystal plane at the level of the membrane. The slice was made approximately
Fig. 1. Several views of the electron density distribution in the twodimensional $\mathrm{H}^{+}$P-ATPase crystals. Density maps at $0.8 \mathrm{~nm}$ resolution (red) and $1.4 \mathrm{~nm}$ resolution (blue) are overlaid. The red map shows the high-density features and the blue map shows the low-resolution envelope. (A) A cross section of the map cut perpendicular to the two-dimensional crystal plane, which is also the membrane plane. The repeating unit of the ATPase crystals is a hexamer made up of six $100 \mathrm{kDa}$ monomers. The vertical arrowheads delineate the density of a single ATPase hexamer. The crystals consist of two offset layers of ATPase hexamers separated by a narrow region of low density. Arrow 1 points to the membraneembedded region of the ATPase in one of the layers. Arrow 2 points to the cytoplasmic region of the ATPase in the same layer. Arrow 3 points to the major protein-protein contact between the layers. (B) A section of the maps cut parallel to the crystal plane through one of the hexamer layers in the membrane-embedded region of the ATPase at a level indicated approximately by arrow 1 in A. (C) A similar section cut through the maps at a level indicated approximately by arrow 2 in $\mathrm{A}$. The arrow in $\mathrm{C}$ indicates a major protein-protein contact in the ATPase crystal lattice. Scale bars, $5 \mathrm{~nm}$. See text for additional details.

through the region indicated by arrow 1 in Fig. 1A. This view shows a striking pattern of circular units tightly packed on a hexagonal lattice, each unit representing an ATPase hexamer. At this level of the map, the hexamers appear as two concentric rings with outer diameters of $11.5 \mathrm{~nm}$ and $16.5 \mathrm{~nm}$, respectively. The inner ring comprises six sets of ten transmembrane helices arranged in a ring around an apparent sixfold symmetry axis, representing the membrane domains of six ATPase monomers. Although the presence of transmembrane helices in the molecule was suspected, their existence and their number were conjectural until the structure was seen.

An unexpected largesse that emerged from the structure is the clear-cut visualization of the organization of the detergent in the ATPase crystals. The outer rings seen in Fig. 1B are the DDM used in the ATPase crystallization system. Cross sections of the detergent rings can be seen in Fig. 1A just outside the transmembrane helix region of each hexamer. The detergent therefore forms toroid rings around the normally membrane-embedded regions of six monomers of the ATPase hexamers, and these rings then bind side to side, and possibly also face to face in certain regions, to form an organized detergent network in the crystals. Detergent-detergent interactions thus appear to provide a substantial contribution to the crystal-packing forces in the ATPase crystals.

Fig. 1C shows a section cut parallel to the crystal plane through the large cytoplasmic domain region that lies above the membrane-spanning region. This slice was made approximately through the region indicated by arrow 2 in Fig. 1A. It shows clearly that protein-protein crystal contacts occur in the cytoplasmic region as well. The predominant crystal contact in this region of the crystals is indicated by the arrow.

Thus, another important piece of information that emerged from the structure is that the crystal contacts comprise both 
protein-protein and detergent-detergent interactions. As mentioned above, the original approach to obtaining the $\mathrm{H}^{+} \mathrm{P}$ ATPase crystals recognized the possible importance of attaining a balance between the solubility of the protein surfaces of the ATPase molecule and the solubility of its detergent micellar collar surrounding the membrane-embedded region. The clear-cut demonstration of both protein-protein and detergent-detergent interactions in the $\mathrm{H}^{+} \mathrm{P}$-ATPase crystal structure lent gratifying credence to the validity of this concept and suggests that it might be useful for obtaining twoand three-dimensional crystals of other membrane proteins as well. In accord with this, the sparse matrix membrane protein crystallization protocols described by Song and Gouaux (1997) are designed to maintain the conditions near the solubility limit of the detergent micelles.

Fig. 2 shows a drawing of the transmembrane helix region of an ATPase monomer, viewed from the cytoplasmic side of the membrane. The transmembrane helix region covers an area of approximately $3 \mathrm{~nm} \times 4 \mathrm{~nm}$ and is $3.5-4 \mathrm{~nm}$ thick, as judged by the average extent of the membrane-spanning helices in the vertical direction. The ten membrane-spanning helices are labelled A-J. The helices are tightly packed, with center-tocenter distances at the points of closest contact ranging from approximately 0.8 to $1.3 \mathrm{~nm}$. None of them runs exactly perpendicular to the membrane plane. The tilt angles of helices $\mathrm{B}, \mathrm{C}, \mathrm{D}, \mathrm{F}, \mathrm{G}$ and $\mathrm{J}$ range from approximately 5 to $15^{\circ}$ whereas helices $\mathrm{A}, \mathrm{E}, \mathrm{H}$ and I are more highly tilted by approximately 16-22 ${ }^{\circ}$. Helices A, B, C and E appear to form a four-helix bundle with a pronounced right-handed twist. Helices D, F, H and $\mathrm{G}$ form a second layer on one side of the bundle, their orientation being similar to that of helices $\mathrm{C}$ and E. Helices I and $\mathrm{J}$, which again have the same orientation, form a third layer. Most helices are essentially straight except helices E and I, which are slightly curved. The apparent lengths of individual membrane-spanning helices vary considerably, but they average approximately $3.5 \mathrm{~nm}$ long, as expected for membrane-spanning helices. The order of the ten transmembrane helices in the linear sequence of the polypeptide chain is unknown.

Fig. 3A shows a surface-shaded representation of a single ATPase monomer viewed along the plane of the membrane. The monomer is the functional unit for this transporter, because we have demonstrated previously that there are no subunits other than the $100 \mathrm{kDa}$ polypeptide chain (Scarborough and Addison, 1984) and that monomers are fully functional in ATP-hydrolysis-driven proton translocation (Goormaghtigh et al., 1986). The arrows indicate the approximate boundaries of the lipid bilayer in the natural milieu of the molecule. As predicted from hydropathy analyses and demonstrated in biochemical studies (Rao et al., 1988; Hennessey and Scarborough, 1990; Scarborough and Hennessey, 1990; Rao et al., 1991, 1992), the $\mathrm{H}^{+}$P-ATPase possesses a large cytoplasmic sector (upper part of Fig. 3A), a membrane sector that constitutes approximately one-quarter of the molecule and a small extracellular portion.

Another important feature of the ATPase molecule revealed
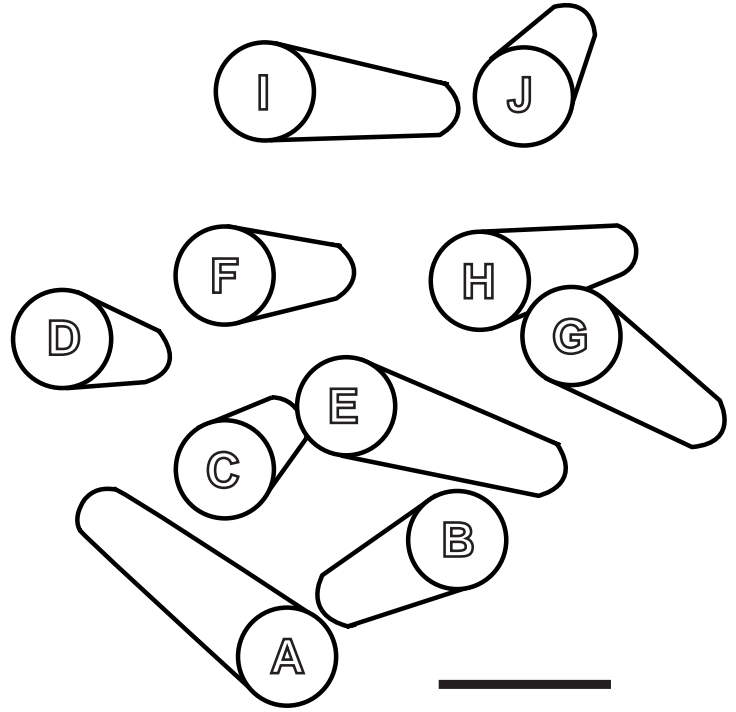

Fig. 2. Drawing of the 10 transmembrane helices of an $\mathrm{H}^{+} \mathrm{P}-\mathrm{ATPase}$ monomer viewed from the cytoplasmic side of the membrane. Each cylinder follows the density of a transmembrane helix in the structure maps shown in Fig. 1. The helices are lettered A-J for reference only; the order of the helices in the linear sequence of the polypeptide chain is unknown. See text for additional details. Bar, $1 \mathrm{~nm}$.

by the structure is the existence in the cytoplasmic region of several separate domains, numbered 1-4 in Fig. 3. Domain 1 is the largest, and it is separated from both domains 3 and 4 by deep clefts. The interdomain separations are best seen in the top view shown in Fig. 3B. Each of domains 1, 3 and 4 is connected to the membrane by extensions of the transmembrane helices. Domain 2 is an extension of domain 1 and is involved in the formation of the hexamers that form when the ATPase is isolated (Chadwick et al., 1987).

\section{Conformational dynamics of the $\mathbf{H}^{+} \mathbf{P}$-ATPase molecule}

Early experiments carried out with isolated Neurospora crassa plasma membranes showed that inhibition of the $\mathrm{H}^{+} \mathrm{P}-$ ATPase by mercurial compounds is suppressed by ATP and that the enzyme is markedly protected against inactivation by trypsin when its substrate, MgATP, is present (Scarborough, 1977). Subsequently, this differential susceptibility of the ATPase to tryptic degradation in the presence or absence of MgATP was used for identifying the $\mathrm{H}^{+}$P-ATPase and demonstrating its phosphorylated intermediate (Dame and Scarborough, 1980). During these studies, it was also observed that the potent $\mathrm{H}^{+} \mathrm{P}$-ATPase inhibitor orthovanadate augments the protective effects of MgATP against tryptic degradation, and in subsequent experiments it was found that MgATP plus orthovanadate confers remarkable stability on the ATPase during its detergent solubilization and purification (Addison and Scarborough, 1981). Collectively, these observations suggested that the ATPase undergoes conformational changes during its catalytic cycle. And because such conformational 

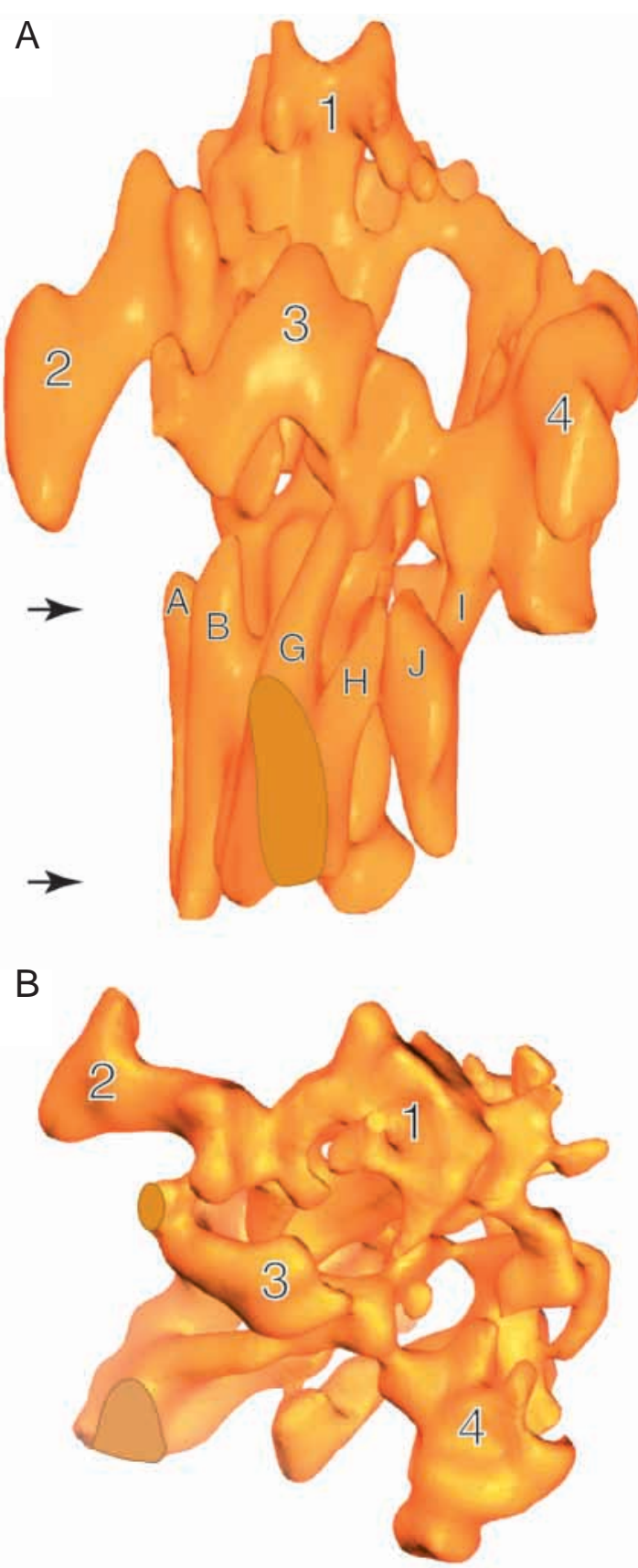

changes are almost certainly an essential feature of the molecular mechanism of ion transport catalyzed by the ATPase, they were investigated in more detail in studies of the effects of a variety of reaction cycle participants or analogues thereof on the rate and pattern of degradation of the ATPase by trypsin (Addison and Scarborough, 1982). Briefly, the results of these experiments showed that, upon binding of MgADP, a substrate analogue, the $\mathrm{H}^{+} \mathrm{P}$-ATPase undergoes a significant conformational change, as shown by a marked increase in resistance to trypsinolysis. Upon binding $\operatorname{Mg}(\beta, \gamma)$ methylene ATP, a transition-state analogue of the enzyme phosphorylation reaction, a similar, but detectably different, conformational change occurs, and binding of $\mathrm{Mg}^{2+}$ and orthovanadate, a transition-state analogue of the enzyme
Fig. 3. Surface-shaded representations of an $\mathrm{H}^{+} \mathrm{P}-\mathrm{ATPase}$ monomer. The images were generated from the structure maps shown in Fig. 1 as described by Auer et al. (1998). (A) A view along the membrane plane. The arrows indicate the approximate boundaries of the membrane in the native milieu of the molecule. The cytoplasmic side is above the membrane plane. The visible helices are labeled as in Fig. 2. (B) A view from the top of the molecule as shown in A. This would be the view from the cytoplasm. The cytoplasmic region of the ATPase molecule comprises several domains, numbered 1-4 in A and B. The clefts between these domains are best seen in the top view (B). See text for additional details.

dephosphorylation reaction, induces a similar conformational change.

As explained in the original report of these findings (Addison and Scarborough, 1982) and elsewhere (Scarborough, 1982, 1985a, 1992; Scarborough and Addison, 1984), the results of these ligand protection experiments made clear the central role of the transition states of the $\mathrm{H}^{+} \mathrm{P}$-ATPase phosphorylation and dephosphorylation reactions in the molecular dynamics of the ATPase reaction cycle. And the importance of the transition states cannot be overemphasized. The fundamental driving force for all enzyme-catalyzed reactions is transition-state binding affinity. As recognized by Pauling (1946) and elaborated upon by others (Jencks, 1966; Wolfenden, 1969; Lienhard, 1973; Fersht et al., 1986), enzymes facilitate chemical reactions by binding most tightly to the transition-state configuration of the reaction that they catalyze. In so doing, they increase the relative concentration or probability of the transition state which, according to the transition-state theory of reaction rates (Frost and Pearson, 1961), leads to rate enhancement because the transition-state concentration is rate-limiting. Implicit in this view of catalysis are numerous favorable bonding interactions between enzyme functional groups and the transition-state configuration of the chemical constituents undergoing the catalyzed reaction. And it is these favorable bonding interactions that drive the protein conformational changes that occur during most, if not all, enzyme-catalyzed reactions (Scarborough, 1982, 1985b, 1992). Normally, the transition state breaks down spontaneously at a great rate, leading to its fleeting existence, an efficient catalytic throughput and a repeating cycle of conformational oscillations. However, in the presence of transition-state analogues such as $\beta, \gamma$-methylene ATP and orthovanadate, which are chemically stable when bound, and substrate analogues such as ADP, which cannot form the transition state, these favorable bonding interactions combine to lock the enzyme in the conformational state appropriate for the particular ligand.

Although it seemed likely that the conformational changes that the ATPase undergoes during its catalytic cycle are substantial, ligand protection of only a few key amino acid residues could conceivably explain the results of the tryptic hydrolysis experiments. The ligand-induced ATPase conformational changes were therefore further explored using attenuated total reflection Fourier transform infrared 
spectroscopy (Goormaghtigh et al., 1994). The results clearly showed that the secondary structure components of the ATPase, i.e. $\alpha$-helix, $\beta$-sheet, turns and random coil, do not change when the molecule undergoes these ligand-induced conformational changes, in agreement with our earlier circular dichroism results (Hennessey and Scarborough, 1988). But importantly, the hydrogen/deuterium (H/D) exchange rates of approximately 175 surface amide linkages in the ATPase polypeptide chain out of a total of approximately 350 are drastically reduced as the ATPase proceeds from its unliganded conformation to its substrate-binding conformation upon binding MgADP. Similarly, the H/D exchange rates of approximately 130 residues are reduced in the transition state of the enzyme dephosphorylation reaction (i.e. bound to $\mathrm{Mg}$ orthovanadate in the presence of ATP). Since virtually all enzymes (Schulz and Schirmer, 1979), particularly those that catalyze phosphoryl-transfer reactions (Steitz et al., 1976; Sachsenheimer and Schulz, 1977; Stuart et al., 1979; Evans and Hudson, 1979; Banks et al., 1979; Pickover et al., 1979; Anderson et al., 1979), possess structures with at least two, discrete, relatively rigid structural domains separated by an often deep cleft that closes during catalysis, these results constitute strong evidence for a similar behavior of the $\mathrm{H}^{+} \mathrm{P}-$ ATPase during its catalytic cycle. Thus, in keeping with these known modes of enzyme conformational dynamics, our results collectively indicate that the ATPase undergoes major rigidbody interdomain movements and one or more resultant cleft closures as it proceeds through its transport cycle.

Referring to the ATPase structure shown in Fig. 3, the probable nature of these conformational transitions during the ATPase catalytic cycle is clear. In the absence of any ligands besides protons, the molecule is in the open conformation shown. Then, in response to the binding of its substrate, MgATP, and progression to the transition state of the enzyme phosphorylation reaction, it is likely that domains 1,3 and 4 move together to close the clefts between them, like the closing of the petals of a flower. Domain 2 presumably does not participate in such motions because it is involved in a major monomer-monomer contact in the hexamers, and monomers in the hexamers retain catalytic function (Hennessey and Scarborough, 1988). It is not known whether the domains move apart again after the completion of the enzyme phosphorylation reaction. But if they do, they clearly must move back together again during the enzyme dephosphorylation reaction, as demonstrated by the occlusion of many surface residues in the presence of MgATP plus orthovanadate (Goormaghtigh et al., 1994). Finally, when the transition state of the enzyme dephosphorylation reaction breaks down, the enzyme reopens to the state shown in Fig. 3 so that it may again bind proton(s) and MgATP, and begin another round of catalysis. Whether the transmembrane helices move in concert with the cytoplasmic domains remains to be seen, but this seems quite likely.

The above view of the conformational dynamics of the $\mathrm{H}^{+}$ P-ATPase molecule during its catalytic cycle is somewhat different from the E1-E2 model for the P-type ATPases that is more commonly invoked (Inesi, 1985). The central feature of the E1-E2 model is a slow conformational interconversion between E1 and E2 states that changes the accessibility and affinity of the ion-binding sites with respect to the membrane surface. In the E1 form, the ion-binding sites are of high affinity and in contact with the cytoplasmic or ATP side of the membrane; in the E2 form, the sites are of low affinity and in contact with the opposite side of the membrane. Phosphorylated forms participate in a similar interconversion between E1-P and E2-P. The forces that drive the conformational changes are rarely specified. A major shortcoming of the E1-E2 model is that it fails to recognize the essential role of transition-state binding affinity in the enzyme phosphorylation and dephosphorylation reactions as the real driving force for the conformational changes that occur during the catalytic cycle of the P-type ATPases. Its heuristic value is therefore questionable. Moreover, the model has not withstood experimental scrutiny. In a trenchant biochemical analysis, Jencks and his colleagues have provided convincing evidence that the conformational transitions inherent in the E1-E2 model probably do not occur (for a review, see Jencks, 1989). Rather, the accessibility and affinity of the ion-binding sites are controlled by the enzyme phosphorylation state, as implied here and explicitly proposed by Scarborough (1982, 1985b). The E1-E2 model is therefore not very useful, somewhat misleading and should be discarded. As a replacement, the simple scheme of Jencks (1989) is appealing, supported by numerous experimental results and probably much closer to reality. It is also completely consistent with the conformational dynamics of the $\mathrm{H}^{+}$P-ATPase molecule proposed above.

\section{Molecular mechanism of ion transport}

The molecular mechanism by which the conformational dynamics described above drives ion translocation against a concentration gradient is, as yet, unknown. And, elucidation of the mechanism will require more detailed information regarding the spatial relationship between the site of aspartate phosphorylation and the ion-binding sites. There is substantial evidence that the ion-binding sites are in the transmembrane helix region (Scott, 1988; Clarke et al., 1989), but the distance from these sites to the active-site aspartate remains controversial, at best. Numerous investigators have concluded that the site of ATP-binding and aspartate phosphorylation is far removed from the ion-binding sites (Highsmith and Murphy, 1984; Scott, 1985; Teruel and Gomez-Fernandez, 1986; Squier et al., 1987; Yonekura et al., 1997). However, others have reached the opposite conclusion (Grisham et al., 1974; Grisham and Mildvan, 1975; Grisham, 1981; Herrmann et al., 1986; Klemens et al., 1988; Klemens and Grisham, 1988; Lutsenko and Kaplan, 1994). Obviously, the means by which the conformational articulations driven by the enzyme phosphorylation and dephosphorylation reactions affect the affinity and accessibility of the ion-binding site could be quite different depending on which of the above possibilities is 
correct. A complete understanding of the molecular mechanism of ATP-hydrolysis-driven ion transport catalyzed by the P-type ATPases must therefore await an atomic resolution structure of one of these fascinating molecular machines.

This work was supported by United States Public Health Service, National Institutes of Health, Grant GM24784.

\section{References}

Addison, R. and Scarborough, G. A. (1981). Solubilization and purification of the Neurospora plasma membrane $\mathrm{H}^{+}$-ATPase. $J$. Biol. Chem. 256, 13165-13171.

Addison, R. and Scarborough, G. A. (1982). Conformational changes of the Neurospora plasma membrane $\mathrm{H}^{+}$-ATPase during its catalytic cycle. J. Biol. Chem. 257, 10421-10426.

Anderson, C. M., Zucker, F. H. and Steitz, T. A. (1979). Spacefilling models of kinase clefts and conformation changes. Science 204, 375-380.

Auer, M., Scarborough, G. A. and Kühlbrandt, W. (1998). Threedimensional map of the plasma membrane $\mathrm{H}^{+}$-ATPase in the open conformation. Nature 392, 840-843.

Auer, M., Scarborough, G. A. and Kühlbrandt, W. (1999). Surface crystallization of the plasma membrane $\mathrm{H}^{+}$-ATPase on a carbon support film for electron crystallography. J. Mol. Biol. 287, 961-968.

Banks, R. D., Blake, C. C. F., Evans, P. R., Haser, R., Rice, D. W., Hardy, G. W., Merrett, M. and Phillips, A. W. (1979). Sequence, structure and activity of phosphoglycerate kinase: a possible hingebending enzyme. Nature 279, 773-777.

Chadwick, C., Goormaghtigh, E. and Scarborough, G. A. (1987) A hexameric form of the Neurospora crassa plasma membrane $\mathrm{H}^{+}-$ ATPase. Arch. Biochem. Biophys. 252, 348-356.

Clarke, D. M., Loo, T. W., Inesi, G. and MacLennan, D. H. (1989). Location of high affinity $\mathrm{Ca}^{++}$-binding sites within the predicted transmembrane domain of the sarcoplasmic reticulum $\mathrm{Ca}^{++}$ ATPase. Nature 339, 476-478.

Cyrklaff, M., Auer, M., Kühlbrandt, W. and Scarborough, G. A. (1995). Two-dimensional structure of the Neurospora crassa plasma membrane ATPase as determined by electron crystallography. EMBO J. 14, 1854-1857.

Dame, J. B. and Scarborough, G. A. (1980). Identification of the hydrolytic moiety of the Neurospora plasma membrane $\mathrm{H}^{+}$-ATPase and demonstration of a phosphoryl-enzyme intermediate in its catalytic mechanism. Biochemistry 19, 2931-2937.

Evans, P. R. and Hudson, P. J. (1979). Structure and control of phosphofructokinase from Bacillus stearothermophilus. Nature 279, 500-504.

Fersht, A. R., Leatherbarrow, R. J. and Wells, T. N. C. (1986). Binding energy and catalysis: a lesson from protein engineering of the tyrosyl-tRNA synthetase. Trends. Biochem. Sci. 11, 321-325.

Frost, A. A. and Pearson, R. G. (1961). Transition-state theory. In Kinetics and Mechanism, second edition, pp. 77-102. New York: John Wiley \& Sons.

Goormaghtigh, E., Chadwick, C. and Scarborough, G. A. (1986). Monomers of the Neurospora plasma membrane $\mathrm{H}^{+}$-ATPase catalyze efficient proton translocation. J. Biol. Chem. 261, 7466-7471.

Goormaghtigh, E., Vigneron, L., Scarborough, G. A. and
Ruysschaert, J.-M. (1994). Tertiary conformational changes of the Neurospora crassa plasma membrane $\mathrm{H}^{+}$-ATPase monitored by hydrogen/deuterium exchange kinetics: a Fourier transform infrared spectroscopy approach. J. Biol. Chem. 269, 27409-27413.

Grisham, C. M. (1981). Characterization of ATP binding sites of sheep kidney medulla $\left(\mathrm{Na}^{+}+\mathrm{K}^{+}\right)$-ATPase using CrATP. J. Inorg. Biochem. 14, 45-57.

Grisham, C. M., Gupta, R. K., Barnett, R. E. and Mildvan, A. S. (1974). Thallium-205 nuclear relaxation and kinetic studies of sodium and potassium ion-activated adenosine triphosphatase. $J$. Biol. Chem. 249, 6738-6744.

Grisham, C. M. and Mildvan, A. S. (1975). Magnetic resonance and kinetic studies of the mechanism of membrane-bound sodium and potassium ion-activated adenosine triphosphatase. J. Supromol. Struct. 3, 304-313.

Hennessey, J. P. and Scarborough, G. A. (1988). Secondary structure of the Neurospora crassa plasma membrane $\mathrm{H}^{+}$-ATPase as estimated by circular dichroism. J. Biol. Chem. 263, 3123-3130.

Hennessey, J. P. and Scarborough, G. A. (1990). Direct evidence for the cytoplasmic location of the $\mathrm{NH}_{2}$ - and $\mathrm{COOH}$-terminal ends of the Neurospora crassa plasma membrane $\mathrm{H}^{+}$-ATPase. J. Biol. Chem. 265, 532-537.

Herrmann, T. R., Gangola, P. and Shamoo, A. E. (1986). Estimation of inter-binding-site distances in sarcoplasmic reticulum $\left(\mathrm{Ca}^{2+}+\mathrm{Mg}^{2+}\right)$-ATPase using $\mathrm{Eu}(\mathrm{III})$ luminescence energy transfer. Eur. J. Biochem. 158, 555-560.

Highsmith, S. and Murphy, A. J. (1984). $\mathrm{Nd}^{3+}$ and $\mathrm{Co}^{2+}$ binding to sarcoplasmic reticulum CaATPase. J. Biol. Chem. 259 , 14651-14656.

Inesi, G. (1985). Mechanism of calcium transport. Annu. Rev. Physiol. 47, 573-601.

Jencks, W. P. (1966). Strain and conformation change in enzymatic catalysis. In Current Aspects of Biochemical Energetics (ed. N. O. Kaplan and E .P. Kennedy), pp. 273-298. New York: Academic Press.

Jencks, W. P. (1989). How does a calcium pump pump calcium? $J$. Biol. Chem. 264, 18855-18858.

Klemens, M. R. and Grisham, C. M. (1988). NMR studies identify four intermediate states of ATPase and the ion transport cycle of sarcoplasmic reticulum $\mathrm{Ca}^{++}$-ATPase. FEBS Lett. 237, 4-8.

Klemens, M. R., Stewart, J. M. MacD., Mahaney, J. E., Kuntzweiler, T. A., Sattler, M. C. and Grisham, C. M. (1988) NMR and ESR studies of active site structures and intermediate states of kidney Na,K-ATPase and SR CaATPase. In Advances in Biotechnology of Membrane Ion Transport (ed. P. L. Jorgensen and R. Verna), pp. 107-124. New York: Raven Press.

Lienhard, G. (1973). Enzymatic catalysis and transition-state theory. Science 180, 149-154.

Lutsenko, S. and Kaplan, J. H. (1994). Molecular events in close proximity to the membrane associated with the binding of ligands to the Na,K-ATPase. J. Biol. Chem. 269, 4555-4564.

Lutsenko, S. and Kaplan, J. (1995). Organization of P-type ATPases: significance of structural diversity. Biochemistry 34, 15607-15613.

McPherson, A. (1982). Preparation and Analysis of Protein Crystals. New York: John Wiley \& Sons.

Moller, J. P., Juul, B. and le Maire, M. (1996). Structural organization, ion transport and energy transduction of P-type ATPases. Biochim. Biophys. Acta 1286, 1-51.

Pauling, L. (1946). Molecular architecture and biological reactions. Chem. Eng. News 24, 1375-1377. 
Pedersen, P. L. and Carafoli, E. (1987). Ion motive ATPases. I. Ubiquity, properties and significance to cell function. Trends Biochem. Sci. 12, 146-150.

Pickover, C. A., McKay, D. B., Engelman, D. M. and Steitz, T. A. (1979). Substrate binding closes the cleft between the domains of yeast phosphoglycerate kinase. J. Biol. Chem. 254, 11323-11329.

Rao, U. S., Bauzon, D. D. and Scarborough, G. A. (1992). Cytoplasmic location of amino acids 359-440 of the Neurospora crassa plasma membrane $\mathrm{H}^{+}$-ATPase. Biochim. Biophys. Acta 1108, 153-158.

Rao, U. S., Hennessey, J. P. and Scarborough, G. A. (1988). Protein chemistry of the Neurospora crassa plasma membrane $\mathrm{H}^{+}$-ATPase. Analyt. Biochem. 173, 251-264.

Rao, U. S., Hennessey, J. P. and Scarborough, G. A. (1991). Identification of the membrane-embedded regions of the Neurospora crassa plasma membrane $\mathrm{H}^{+}$-ATPase. J. Biol. Chem. 266, 14740-14746.

Sachsenheimer, W. and Schulz, G. E. (1977). Two conformations of crystalline adenylate kinase. J. Mol. Biol. 114, 23-36.

Scarborough, G. A. (1977). Properties of the Neurospora crassa plasma membrane ATPase. Arch. Biochem. Biophys. 180, 384-393.

Scarborough, G. A. (1982). Chemiosmotic models for the mechanisms of the cationmotive ATPases. Ann. N.Y. Acad. Sci. 402, 99-115.

Scarborough, G. A. (1985a). The mechanisms of energization of solute transport in fungi. In Environmental Regulation of Microbial Metabolism (ed. I. S. Kulaev, E. A. Dawes and D. W. Tempest), pp. 39-51. London: Academic Press.

Scarborough, G. A. (1985b). Binding energy, conformational change and the mechanism of transmembrane solute movements. Microbiol. Rev. 49, 214-231.

Scarborough, G. A. (1992). The Neurospora crassa plasma membrane $\mathrm{H}^{+}$-ATPase. In New Comprehensive Biochemistry: Molecular Aspects of Transport Proteins (ed. J .J. H. M. de Pont and E .M. Wright), pp. 117-134. Amsterdam: Elsevier Science Publishers B.V.

Scarborough, G. A. (1994). Large single crystals of the Neurospora crassa plasma membrane $\mathrm{H}^{+}$-ATPase: an approach to the crystallization of integral membrane proteins. Acta Cryst. D 50, 643-649.
Scarborough, G. A. and Addison, R. (1984). On the subunit composition of the Neurospora plasma membrane $\mathrm{H}^{+}$-ATPase. $J$. Biol. Chem. 259, 9109-9114.

Scarborough, G. A. and Hennessey, J. P., Jr (1990). Identification of the major cytoplasmic regions of the Neurospora crassa plasma membrane $\mathrm{H}^{+}$-ATPase using protein chemical techniques. J. Biol. Chem. 265, 16145-16149.

Schulz, G. E. and Schirmer, R. H. (1979). Principles of Protein Structure. New York: Springer-Verlag.

Scott, T. L. (1985). Distances between the functional sites of the $\left(\mathrm{Ca}^{2+}+\mathrm{Mg}^{2+}\right)$-ATPase of sarcoplasmic reticulum. J. Biol. Chem. 260, 14421-14423.

Scott, T. L. (1988). Molecular topography of the $\mathrm{Ca}^{++}$-ATPase of sarcoplasmic reticulum. Mol. Cell. Biochem. 82, 51-54.

Song, L. and Gouaux, J. E. (1997). Membrane protein crystallization: application of sparse matrices to the $\alpha$-hemolysin heptamer. Meth. Enzymol. 276, 60-74.

Squier, T. C., Bigelow, D. J., de Ancos, J. G. and Inesi, G. (1987). Localization of site-specific probes on the Ca-ATPase of sarcoplasmic reticulum using fluorescence energy transfer. J. Biol. Chem. 262, 4748-4754.

Stangeland, B., Fugslang, A. T., Malmstrom, S., Axelsen, K. B., Baunsgaard, L., Lanfermeijer, F. C., Venema, K., Okkels, F. T., Askerlund, P. and Palmgren, M. G. (1997). P-type $\mathrm{H}^{+}$- and $\mathrm{Ca}^{2+}$-ATPases in plant cells. Ann. N.Y. Acad. Sci. 834, 77-87.

Steitz, T. A., Fletterick, R. J., Anderson, W. F. and Anderson, C. M. (1976). High resolution x-ray structure of yeast hexokinase, an allosteric protein exhibiting a non-symmetric arrangement of subunits. J. Mol. Biol. 104, 197-222.

Stuart, D. I., Levine, M., Muirhead, H. and Stammers, D. K. (1979). Crystal structure of cat muscle pyruvate kinase at a resolution of 2 Å. J. Mol. Biol. 134, 109-142.

Teruel, J. A. and Gomez-Fernandez, J. C. (1986). Distances between the functional sites of sarcoplasmic reticulum $\left(\mathrm{Ca}^{2+}+\mathrm{Mg}^{2+}\right)$-ATPase and the lipid/water interface. Biochim. Biophys. Acta 863, 178-184.

Wolfenden, R. (1969). Transition state analogues for enzyme catalysis. Nature 223, 704-705.

Yonekura, K., Stokes, D. L., Sasabe, H. and Toyoshima, C. (1997). The ATP-binding site of $\mathrm{Ca}^{++}$-ATPase revealed by electron image analysis. Biophys. J. 72, 997-1005. 